\title{
Alveolar macrophage secretion of vesicular SOCS3 represents a platform for lung cancer therapeutics
}

\author{
Jennifer M. Speth, ${ }^{1}$ Loka R. Penke, ${ }^{1}$ Joseph D. Bazzill, ${ }^{2}$ Kyung Soo Park, ${ }^{3}$ Rafael Gil de Rubio, ${ }^{1}$ \\ Daniel J. Schneider, ${ }^{1}$ Hideyasu Ouchi, James J. Moon, ${ }^{2,3,4}$ Venkateshwar G. Keshamouni, ${ }^{1,4}$ \\ Rachel L. Zemans,' Vibha N. Lama,' Douglas A. Arenberg,' and Marc Peters-Golden ${ }^{1,4}$ \\ 'Division of Pulmonary and Critical Care Medicine, Department of Internal Medicine, University of Michigan Medical School, \\ Ann Arbor, Michigan, USA. 'Department of Pharmaceutical Sciences, University of Michigan College of Pharmacy, Ann \\ Arbor, Michigan, USA. ${ }^{3}$ Department of Biomedical Engineering, University of Michigan College of Engineering, Ann Arbor, \\ Michigan, USA. ${ }^{4}$ Graduate Program in Immunology, University of Michigan Medical School, Ann Arbor, Michigan, USA.
}

\begin{abstract}
Lung cancer remains the leading cause of cancer-related death in the United States. Although the alveolar macrophage (AM) comprises the major resident immune cell in the lung, few studies have investigated its role in lung cancer development. We recently discovered a potentially novel mechanism wherein AMs regulate STAT-induced inflammatory responses in neighboring epithelial cells (ECs) via secretion and delivery of suppressors of cytokine signaling 3 (SOCS3) within extracellular vesicles (EVs). Here, we explored the impact of SOCS3 transfer on EC tumorigenesis and the integrity of AM SOCS3 secretion during development of lung cancer. AM-derived EVs containing SOCS3 inhibited STAT3 activation as well as proliferation and survival of lung adenocarcinoma cells. Levels of secreted SOCS3 were diminished in lungs of patients with nonsmall cell lung cancer and in a mouse model of lung cancer, and the impaired ability of murine AMs to secrete SOCS3 within EVs preceded the development of lung tumors. Loss of this homeostatic brake on tumorigenesis prompted our effort to "rescue" it. Provision of recombinant SOCS3 loaded within synthetic liposomes inhibited proliferation and survival of lung adenocarcinoma cells in vitro as well as malignant transformation of normal ECs. Intratumoral injection of SOCS3 liposomes attenuated tumor growth in a lung cancer xenograft model. This work identifies AMderived vesicular SOCS3 as an endogenous antitumor mechanism that is disrupted within the tumor microenvironment and whose rescue by synthetic liposomes can be leveraged as a potential therapeutic strategy for lung cancer.
\end{abstract}

Conflict of interest: US Patent application 16/071,290 describing these liposomes was filed on July 19 , 2018, byMPG; liposomes are referring to "synthetic liposomes encapsulating recombinant SOCS3."

Copyright: () 2019, American Society for Clinical Investigation.

Submitted: June 26, 2019 Accepted: September 12, 2019 Published: October 17, 2019

Reference information: /CI Insight. 2019;4(20):e131340. https://doi.org/10.1172/jici. insight.131340.

\section{Introduction}

Lung cancer is the leading cancer killer in both men and women, with a devastating 5-year survival rate of approximately $18 \%$. Non-small cell lung cancer (NSCLC) is the most common histologic type. For many reasons, including slow uptake of screening protocols and lack of clinical symptoms in early-stage disease, most patients with NSCLC are diagnosed with late-stage disease with an attendant poor prognosis. Conventional chemotherapy has limited effectiveness. Although new immunomodulatory strategies exhibit promise in NSCLC, limitations are also emerging (1). Clearly, greater insight into the lung tumor microenvironment and antitumor immunity are of utmost importance for developing alternative approaches to treatment.

Numerous studies have implicated chronic inflammation and constitutive activation of STAT proteins, particularly STAT3, in the development and progression of tumors of various organs, including the lung (2-4). A family of prototypic endogenous STAT inhibitors called suppressors of cytokine signaling (SOCS) proteins - exemplified by SOCS3 - are deleted or epigenetically downregulated in many types of solid tumors, and their functional deficiency is implicated in tumor development and metastases $(5,6)$. Indeed, overexpression of SOCS3 in NSCLC cell lines inhibited tumor cell function, suggesting that loss of SOCS3 is critical for tumorigenesis (7). Recently, intratumoral administration of a SOCS3-derived peptide has been reported to inhibit growth of squamous cell carcinoma (8). These studies strongly suggest that SOCS3 expression within tumor cells is an important endogenous brake on tumor formation, and strategies to overcome its downregulation are expected to have antitumor potential. 
The alveolar macrophage (AM) is the major resident immune cell of the pulmonary alveolus and plays a pivotal role in the maintenance of homeostasis in the unique and highly challenging environment of the lung. Because the lung is continuously exposed to inhaled microbes, toxins, and antigens, inflammatory responses in the alveoli must be tightly controlled to ensure preservation of gas exchange. The crosstalk between resident AMs and the epithelial cells (ECs) that comprise the surface of the lung - the source of most lung tumors $(9,10)$ - is essential in maintenance of alveolar homeostasis. Recently, we uncovered a potentially novel role for AMs in restraining inflammatory responses in the lung via the constitutive secretion of SOCS3 within extracellular vesicles (EVs); these EVs are internalized by respiratory ECs, wherein they inhibit STAT activation in response to cytokines, such as IL-6 $(11,12)$. While resident AM functions such as phagocytosis and cytokine secretion are altered in lung cancer patients (13), existing data on the role of the resident AM in lung cancer development and progression are limited and contradictory (14). Here, we identify a potential of AMs to provide exogenous SOCS3 secreted in EVs to ECs. This acquired SOCS3 can inhibit STAT3 activation, proliferation, and survival in adenocarcinoma cells. We further demonstrate that the homeostatic provision of SOCS3 by AMs becomes dysregulated during lung tumorigenesis, and we introduce a therapeutic strategy by which "rescuing" impaired AM secretion of SOCS3 via the administration of synthetic liposomes encapsulating recombinant SOCS3 serves to inhibit EC transformation and expansion in vitro and xenograft tumor growth in vivo.

\section{Results}

Tumor-promoting properties of adenocarcinoma cells are altered by delivery of AM-derived EVs containing SOCS3. In previous studies, we have shown that AM-derived EVs (AM EVs), which contain SOCS3 among a variety of other uncharacterized cargo, can be internalized by, and inhibit cytokine-induced STAT3 activation in, normal lung ECs (11). Lung cancer cells are typically derived from either bronchial or alveolar ECs (AECs) (15), and we therefore wished to determine if EVs exerted similar effects in lung cancer cells. The adenocarcinoma cell line A549 has been shown to have elevated basal levels of pSTAT3 (a measure of activated STAT3) and has demonstrated reduced functions such as proliferation and metastasis in response to STAT3 silencing (16). Therefore, we chose to utilize this cell line in our studies. First, we evaluated the capacity of A549 cells to take up vesicles. A549 cells and L2 cells (a noncancerous type I-like lung AEC line) were incubated for 1 hour with either medium alone (control) or AM EVs that had been labeled with the fluorescent dye PKH26. The cells were washed, and their uptake was determined by flow cytometry. A549 cells robustly took up EVs (as demonstrated by an increase in fluorescence), and their ability to do so actually exceeded that of noncancer AECs (Figure 1A). SOCS3 has been reported to be epigenetically downregulated in lung cancer cells, including A549 (17, 18); thus, these cells represent an appropriate model for investigating the effects of intercellular transfer of AM EVs previously shown to contain SOCS3 (11). We therefore assessed the capacity of AM EVs to inhibit cytokine-induced STAT3 activation in A549 cells. After pretreatment with AM EVs for 2 hours, levels of pSTAT3 (a measure of activated STAT3) in response to treatment with the cytokine IL-6 were significantly lower than in stimulated cells that received no EVs (Figure 1, B and C). Since activated STAT3 is a critical driver of tumor cell proliferation and survival, we next explored the ability of AM EVs to alter these properties of tumor cells. A549 cells were thus treated with AM EVs at a typical $\mathrm{EV} /$ cell ratio of 16:1 for 72 hours, and their intracellular DNA content (a surrogate measure of cell number) was measured using the CyQuant DNA-binding assay. AM EVs significantly reduced A549 cell DNA content compared with untreated controls, suggesting an inhibition of proliferative ability (Figure 1D). Next, we measured the effects of this same number of AM EVs on apoptosis by quantifying via flow cytometry the frequency of A549 cells expressing surface annexin V - a marker of apoptosis - after 24 hours of AM EV exposure. A significantly greater proportion of A549 cells expressed surface annexin V after treatment with AM EVs than did untreated controls. Notably, the magnitude of annexin V expression in response to AM EVs was similar to that of cells treated with the prototypic apoptotic stimulus Fas ligand (FasL) (Figure 1E).

In addition to reflecting decreased proliferation, the reduction in basal DNA binding (Figure 1D) elicited by treatment with AM EVs might also reflect a contribution from increased apoptosis, since these 2 cellular properties often coincide. To distinguish the contributions of decreased cell proliferation vs. increased apoptosis in this assay, we assessed the effects of addition of the pan-caspase inhibitor Z-VAD-FMK prior to treatment with EVs on cellular DNA content of A549 cells as determined by the CyQuant assay. Both by itself and in the presence of FasL, the caspase inhibitor significantly increased cellular DNA content, demonstrating that this "proliferation" assay indeed is also sensitive to changes in apoptosis. Caspase inhibition with Z-VAD-FMK 
A

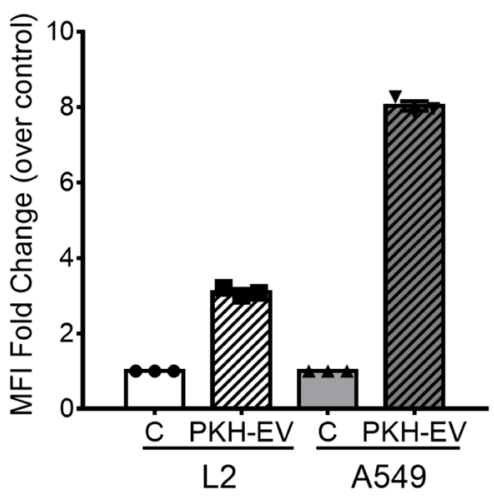

D

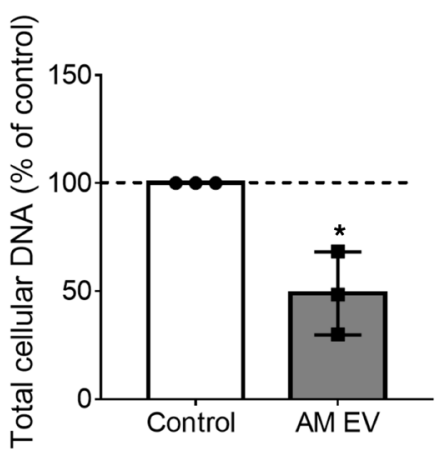

B

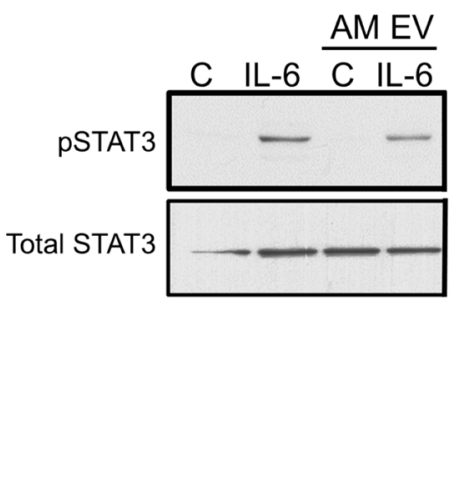

E

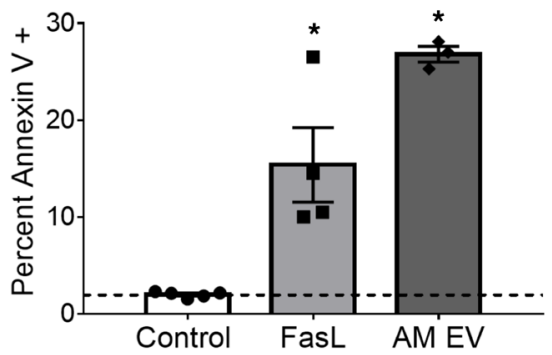

C

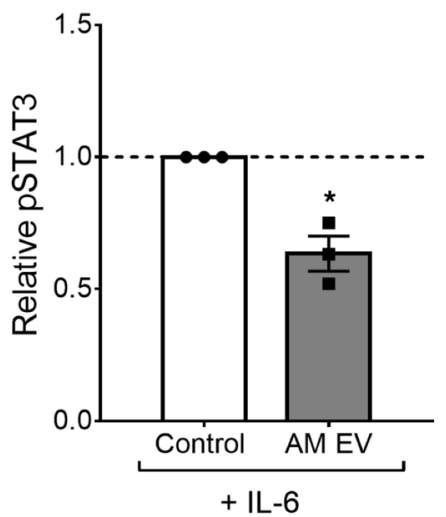

$\mathbf{F}$

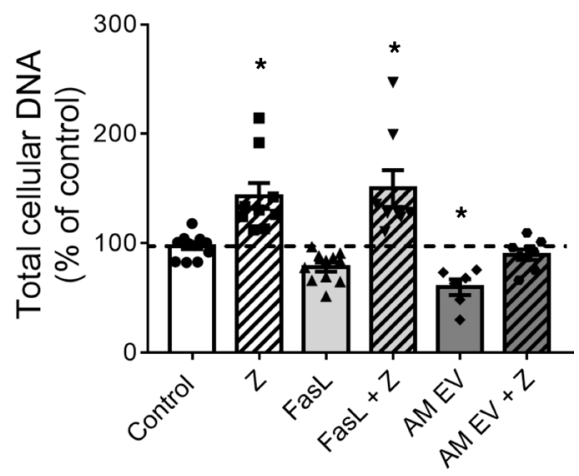

Figure 1. AM-derived EVs inhibit tumor cell function. (A) Normal AECs (L2) or A549 adenocarcinoma cells were incubated with either medium alone (control, C) or PKH26-labeled AM-derived EVs for 1 hour (h); EV uptake was determined by flow cytometry and expressed as mean fluorescence intensity (MFI) ( $n=3$ separate experiments). (B and C) Cells were pretreated with EVs for $2 \mathrm{~h}$, washed, and stimulated with $10 \mathrm{ng} / \mathrm{ml} \mathrm{IL}-6$ for 30 minutes, and lysates were analyzed for pSTAT3 and total STAT3 by WB ( $n=3$ separate experiments). A representative WB (B) and densitometric analysis of multiple such experiments, represented as relative pSTAT3 protein (C), are shown. (D) A549 cells were incubated with AM EVs for $72 \mathrm{~h}$ and subjected to CyQuant cell proliferation assay measuring total cellular DNA ( $n=3$ separate experiments). (E) A549 cells were treated with FasL (as a positive control) or AM EVs for $24 \mathrm{~h}$, and apoptotic cells were determined by annexin $V^{+}$staining via flow cytometry ( $n=3-5$ replicates). (F) A549 cells were treated with AM EVs or FasL for $72 \mathrm{~h}$, with or without the pan-caspase inhibitor Z-VAD-FMK (Z), and cell proliferation was determined using CyQuant assay ( $n=6-14$ replicates from 3 separate experiments). Student's 2-tailed $t$ test was used for comparison of 2 groups (C and $\mathbf{D}$ ) or 1-way ANOVA with Bonferroni's post hoc analysis was used for multiple comparisons ( $\mathbf{E}$ and $\mathbf{F}$ ); ${ }^{*} P<0.05$.

only partially reversed the reduction in DNA content elicited by AM EVs (Figure 1F), suggesting that the actions of EVs reflected both inhibition of proliferation, as well as enhanced apoptosis.

Secreted SOCS3 protein is reduced in the lungs of NSCLC patients. Since SOCS3 protein lacks a leader sequence that would permit conventional secretion, its secretion depends on packaging within EVs. We previously demonstrated that AECs themselves are incapable of secreting SOCS3, indicating that AM EVs are the predominant source of SOCS3 in the alveolar compartment in vivo (11). This process of AM SOCS3 secretion within the lung can be monitored by detection of this protein in bronchoalveolar lavage fluid (BALF) $(11,12$ ). We therefore sought to evaluate the integrity of this homeostatic process in patients with lung cancer. Because SOCS3 protein is sequestered within the interior of EVs, its antibody recognition requires membrane disruption (11), which we accomplished by sonicating the BALF prior to analysis. BALF samples from patients undergoing diagnostic bronchoscopy for pulmonary nodules or masses suspicious for lung cancer were analyzed for SOCS3 via ELISA (Figure 2A). Of a total of 22 patients that were ultimately diagnosed with NSCLC -11 were diagnosed with squamous cell carcinoma, and 11 were diagnosed with adenocarcinoma. Compared with a cohort of healthy volunteers with similar demographics and smoking history (Table 1) and with no known underlying inflammatory or malignant disease, SOCS3 levels in the BALF of NSCLC patients were significantly reduced (Figure 2B). This reduction was independent of histopathologic type (adeno vs. squamous; data not shown), as well as smoking status (former, current, or never smoker). It remained statistically significant, even when the 3 normal subjects with the highest levels of SOCS3 were excluded. 
A

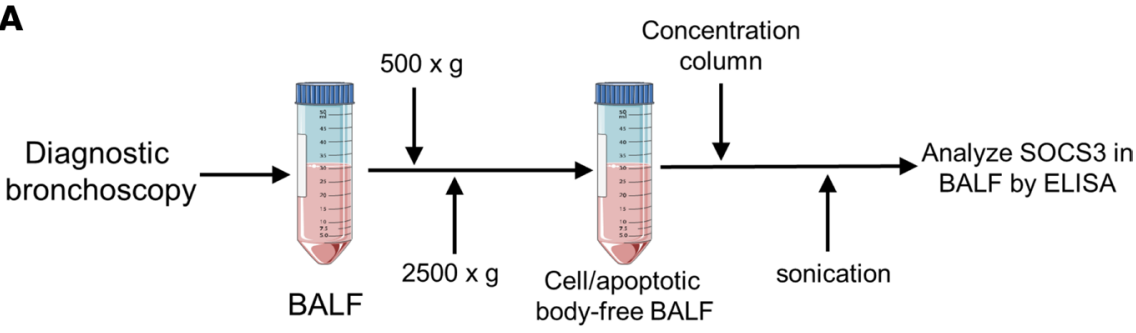

в

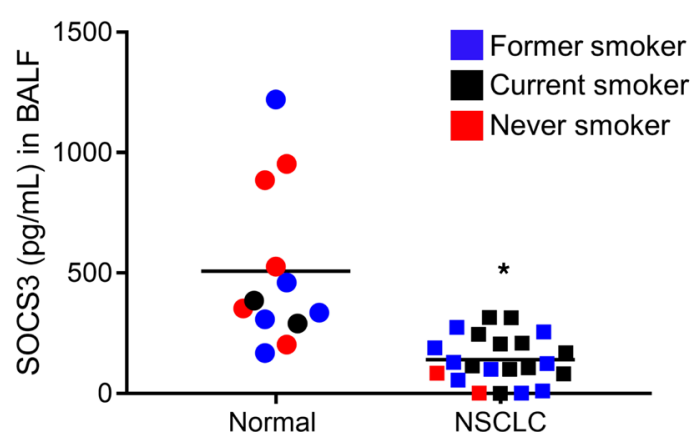

Figure 2. Secreted SOCS3 is reduced in the lungs of NSCLC patients. (A) Schematic depiction of BALF processing for SOCS3 ELISA. (B) Levels of secreted SOCS3 in patient BALF. Data points represent individual patients; horizontal bars represent mean values ( $n=12-22$ patients). Two groups are still significantly different even if 3 normal subjects with highest SOCS3 levels are excluded. Students 2 -tailed $t$ test was used for comparison of 2 groups; ${ }^{*} P<0.05$.

Secreted SOCS3 protein is reduced in the lungs of Kras mutant mice. To confirm, and to investigate the temporal nature of, the relationship between reduced SOCS3 secretion and lung cancer, as observed in patients, we employed a well-established mouse model of mutant Kras-driven adenocarcinoma (19). In this model, G12D mutant Kras expression in the lung is initiated by intrapulmonary delivery of adenoviral Cre recombinase. Tumors arise via a sequence of progression from grade 1 atypical adenomatous hyperplastic lesions visible at 2-4 weeks, to grade 2 adenomas at 6-8 weeks, to grade 3 and visibly detectable adenocarcinoma tumors at approximately 16 weeks (19). Figure 3A illustrates the model, as well as representative histopathologic images of the evolving lesions from harvested mice. As early as 4 weeks after tumor initiation, mutant Kras mice exhibited significantly less secreted SOCS3 protein in their BALF than did WT mice (Figure 3, B and C). SOCS3 levels declined further over time, despite an increase in the numbers of total cells (Figure 3D) and resident AMs (Figure 3E) retrieved by lavage. Since it has been demonstrated that global intrapulmonary adenoviral infection of mutant Kras mice can transduce resident AMs with Kras and alter their phagocytic function (20), we employed 2 approaches to determine whether the reduction in AM SOCS3 secretion in the mouse model was the direct consequence of AM expression of mutant Kras. First, primary rat AMs were placed into culture and transformed with retroviral human mutant KRAS G12V (functionally similar to G12D) and cultured for 1 week. Although these AMs manifested high expression levels of mutant KRAS

Table 1. NSCLC patient demographics

\begin{tabular}{ccc}
\hline Patient demographics & Normal & NSCLC \\
\hline Sample size $(\boldsymbol{n}):$ & 12 & 22 \\
\hline Median age: & 52 years & $48-88$ years \\
\hline Age range: & $23-71$ years & 47 \\
\% Male: & 45 & 88 \\
\% Smoker: & 58 & 9 \\
Former $(\boldsymbol{n}):$ & 5 & 11 \\
\hline Current $(\boldsymbol{n}):$ & 2 & \\
\hline
\end{tabular}




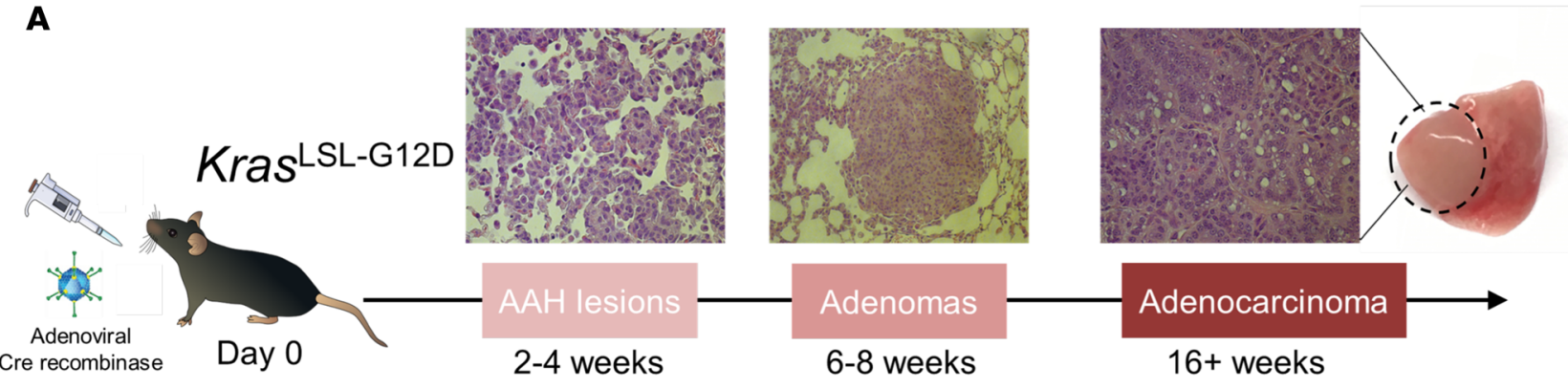

B

BALF

C

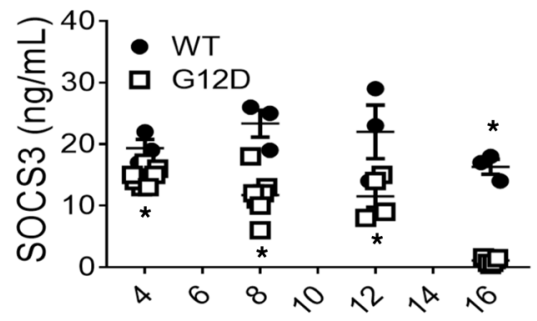

Weeks post AdCre infection

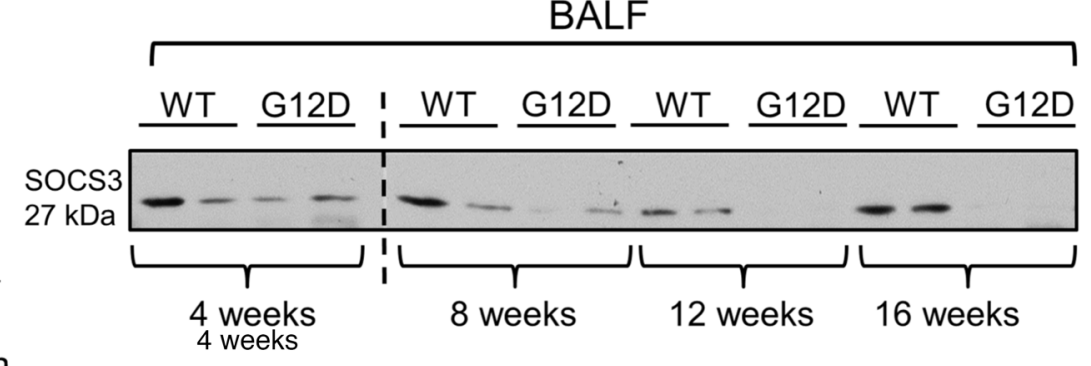

E

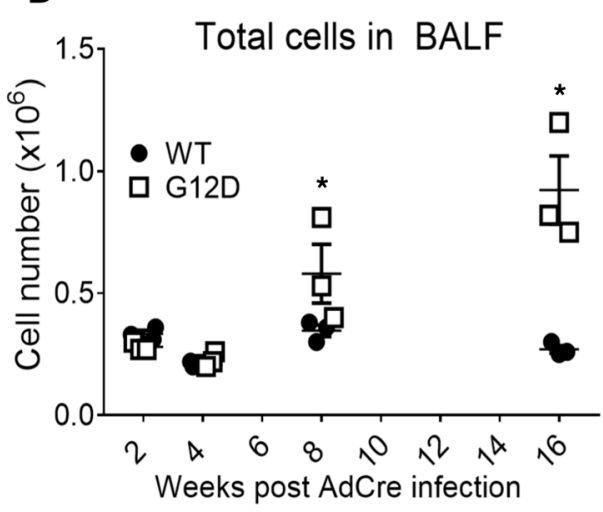

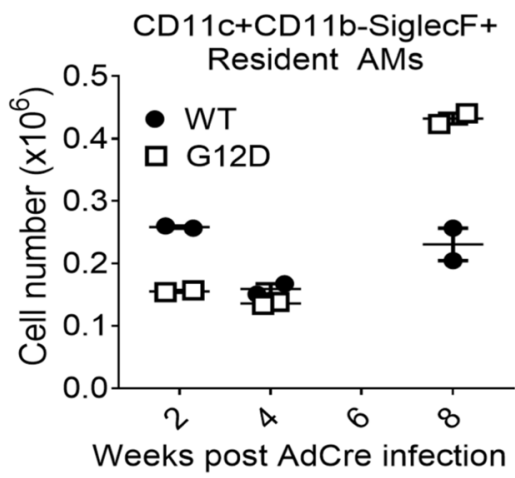

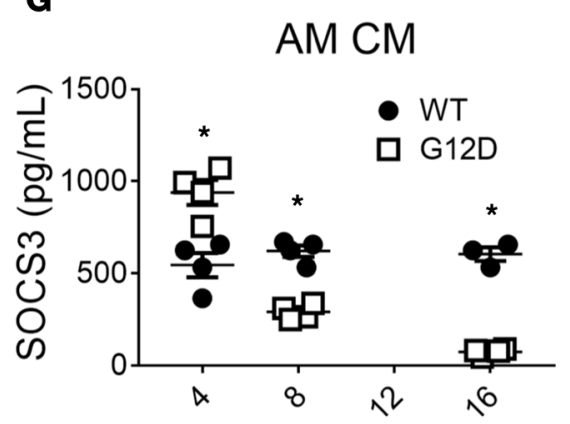

Weeks post AdCre infection
H

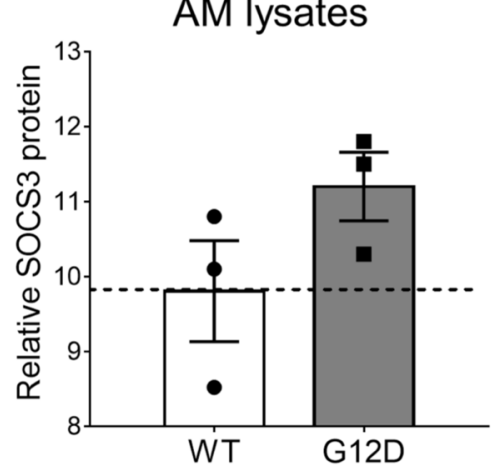

$\mathbf{F}$

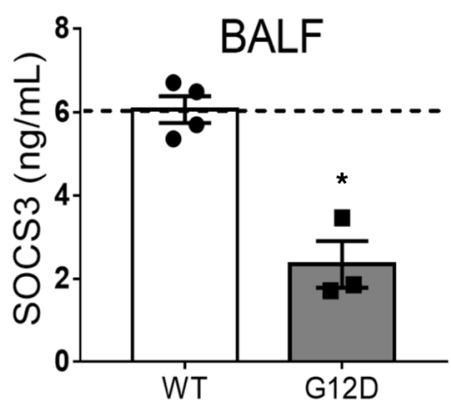

10 weeks post SPC-AdCre

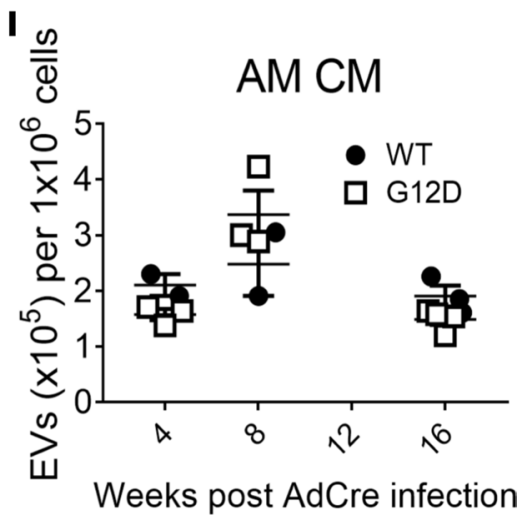

Figure 3. AM packaging of SOCS3 into EVs is reduced in the lungs of Kras mutant mice. (A) Experimental scheme: WT and LSL-Kras ${ }^{\mathrm{C} 12 \mathrm{D}}$ mice were inoculated oropharyngeally with adenoviral Cre recombinase on day 0 . BALF was harvested at 4, 8, 12, and 16 weeks after tumor initiation and analyzed for secreted SOCS3 as well as cell counts; alternatively, AMs were purified by adherence for analysis of their CM. Representative histopathologic lesions from mice harvested at the indicated time points are shown. AAH, atypical adenomatous hyperplasia. (B) SOCS3 concentrations as determined by ELISA in BALF harvested at indicated time points from WT and C12D mice ( $n=3-7$ mice per group; each symbol represents a value from an individual mouse). (C) Representative WB for SOCS3 of BALF samples collected from WT and Kras mutant mice at 4, 8, 12, and 16 weeks after tumor initiation. Each lane represents a sample from an individual mouse $(n=2$ mice). Lanes on either side of the dashed line were from the same blot but were noncontiguous. (D) Numbers of total lavageable cells in the BALF of WT and Kras mutant mice at varying times after tumor initiation ( $n=3$ mice). (E) Numbers of resident 
AMs (defined as CD11 ${ }^{+}$CD11b-Siglec- $F^{+}$cells) in the BALF of WT and Kras mutant mice at various times after tumor initiation ( $n=2$ groups of cells pooled from 2 individual mice). (F) At 10 weeks after tumor initiation with SPC (alveolar EC targeted) specific adenoviral Cre recombinase, BALF was harvested and analyzed for SOCS3 by ELISA ( $n=3-4$ mice per group). (G) Concentration of SOCS3 in CM of AMs isolated from WT and Kras mutant mice at the indicated times after tumor initiation. Cells isolated from the BALF of individual mice of each strain (4 mice per group) were pooled and then divided into triplicate samples. Cells were plated at a concentration of $1 \times 10^{6}$ cells $/ \mathrm{ml}$ for $\sim 20 \mathrm{~h}$. The resulting CM was sonicated and analyzed for SOCS3 by ELISA; data are mean of 3-4 samples. (H) Relative SOCS3 protein levels determined by WB of lysates of AMs obtained at 16 weeks after tumor initiation; note that these are from the same cell cultures whose CM was utilized for ELISA determination in $\mathbf{G}(n=3)$. (I) EV numbers determined in AM CM via flow cytometric analysis and expressed as EV number per $1 \times 10^{6} \mathrm{AMs}$; data are mean of triplicate samples. Student's 2-tailed $t$ test was used for comparison of 2 groups (F and $\mathbf{H}$ ), or 1-way ANOVA with Bonferroni's post hoc analysis was used for multiple comparisons $(\mathbf{B}, \mathbf{D}, \mathbf{E}, \mathbf{G}$, and $\mathbf{I}) ;{ }^{*} P<0.05$.

mRNA, neither intracellular SOCS3 expression nor SOCS3 secretion was affected (Supplemental Figure 1; supplemental material available online with this article; https://doi.org/10.1172/jci.insight.131340DS1). Second, to evaluate this question in vivo, Kras mutant mice were infected with an adenoviral Cre specifically targeting the AEC surfactant protein C promoter (SPC-Ad5Cre), which is not expected to activate mutant Kras in AMs (15). These mice exhibited reduced SOCS3 secretion in their lungs at 10 weeks after tumor initiation (Figure 3F), similar to mice infected with global adenoviral Cre (Figure 3, B and C). Together, these data suggest that reduced SOCS3 protein secretion by AMs in this mouse model of lung cancer is the consequence of the aberrant activation by adenoviral Cre of mutant Kras in the ECs - and not in the AMs themselves. To determine whether reduced SOCS3 secretion by AMs was a cell-intrinsic phenomenon or the consequence of reversible microenvironmental cues in the tumor-bearing lung, resident AMs were retrieved from Kras and WT mice at various times and cultured ex vivo. Resident AMs obtained from Kras mice at 8 and 16 weeks exhibited a reduced capacity to secrete SOCS3 protein during ex vivo culture (Figure $3 G$ ). These differences in secretion were not attributable to declines in intracellular levels of SOCS3 (Figure $3 \mathrm{H})$. To address the possibility that decreased SOCS3 secretion reflected a reduction in the number of total EVs secreted by AMs from Kras mutant mice, EVs were isolated from AM conditioned medium (CM) and enumerated via flow cytometry. The number of such EVs secreted by AMs was similar between WT and Kras mice, suggesting that the decrease in SOCS3 secretion was not due to reduced EV numbers (Figure 3I) but rather to reduced SOCS3 protein loading or packaging within EVs. Likewise, when packaging was expressed as a ratio of SOCS3 protein per secreted vesicle, Kras mutant mice had significantly less $(P<0.05)$ EV-packaged SOCS3 than WT mice at $8(0.18 \pm 0.01$ vs. $0.98 \pm 0.05)$ and $16(0.1 \pm 0.01$ vs. $1.23 \pm 0.07)$ weeks after adenoviral Cre infection. These data demonstrate that the reduction in SOCS3 secretion by AMs in the tumor-bearing lung reflects its impaired packaging within secreted EVs.

Generation and validation of synthetic liposomes containing recombinant SOCS3. The reduction in SOCS3 secretion into BALF observed in both patients with NSCLC and mutant Kras mice during development of adenocarcinomas would be expected to predispose ECs to STAT3 activation and, hence, tumorigenesis. We therefore wished to test the possibility that "rescue" of diminished SOCS3 secretion by AMs via its exogenous provision could exert antitumor actions. Although natural AM-derived EVs contain SOCS3, which we previously implicated in the transcellular inhibition of STAT3 activation in recipient ECs (11), they also are expected to contain a myriad of other cargo molecules of protein, nucleic acid, and lipid composition. In order to mimic SOCS3-containing EVs but eliminate the potential confounding effects of cargo other than SOCS3, we utilized a membrane extrusion technique to generate synthetic phospholipid liposomes encapsulating recombinant SOCS3 protein as their sole cargo (Figure 4A). Liposomes containing either SOCS3 or buffer alone (empty liposomes) had similar size ( $110 \mathrm{~nm}$ diameter), polydispersity index (PDI; indicating a single homogenous population of liposomes), and zeta potential (ZP; indicating a slight negative charge) (Figure 4B and Table 2). For in vitro dosing optimization, serial dilutions of SOCS3 liposomes were compared with the amount of vesicular SOCS3 protein released by $1 \times 10^{6} \mathrm{AMs}$ (the concentration capable of inhibiting STAT3 activation in normal AECs and in previous experiments with A549 cells; Figure 1 ; ref. 11). A total of $10 \mathrm{ng}$ of recombinant SOCS3 protein was determined to yield the bioequivalent amount contained in natural AM EVs from $1 \times 10^{6}$ cells (Figure 4C). To test whether this dose of SOCS3 liposomes was functionally capable of inhibiting activation of STAT3 in cancer cells, A549 cells were pretreated with either SOCS3 liposomes or an equal volume of empty liposomes prior to activation with IL-6. Treatment of A549 cells with this amount of SOCS3-containing liposomes resulted in a significant reduction in pSTAT3 protein (Figure 4D), reflecting a similar degree of inhibition as that observed for treatment with naturally derived AM EVs (Figure 1, B and C). In all subsequent studies, $10 \mathrm{ng}$ SOCS3 liposomes (or equal volume of empty liposomes) was added per well of recipient cells. 
A
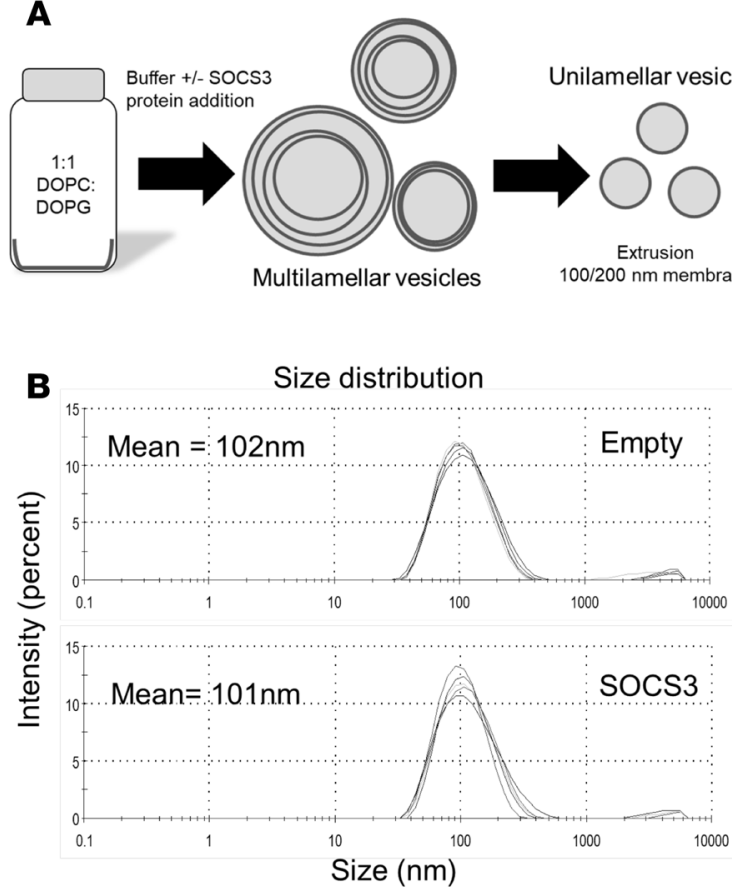

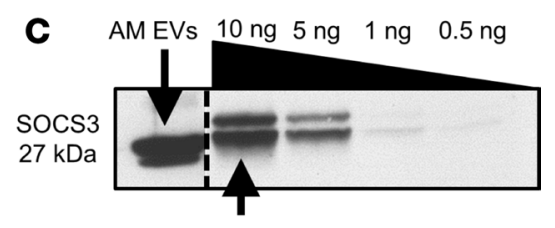

Synthetic liposomes

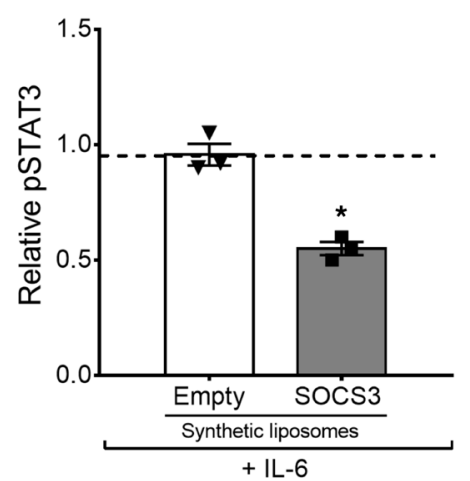

Figure 4. Generation of and characterization of recombinant SOCS3-containing liposomes. (A) Schematic of liposome generation. (B) Histogram: Size distribution of both empty and SOCS3-containing liposomes from 1 representative liposome preparation. (C) Representative WB comparing relative SOCS3 protein amounts in EVs isolated from $1 \times 10^{6} \mathrm{AMs}$ vs. serial dilutions of synthetic SOCS3-containing liposomes. (D) A549 cells were pretreated with liposomes containing $10 \mathrm{ng}$ of SOCS3 protein, or an equal volume of empty liposomes containing only PBS, for $1 \mathrm{~h}$. They were then washed and stimulated with $10 \mathrm{ng} / \mathrm{ml} \mathrm{IL-6}$ for 30 minutes, and lysates were probed for pSTAT3. Top: Representative WB; bottom: mean densitometric analysis of 3 separate experiments, represented as relative PSTAT3 protein. Dashed line denotes noncontiguous lanes from the same blot. pSTAT3 and total STAT3 were from separate gels/blots. Student's 2-tailed $t$ test was used for comparison of 2 groups; ${ }^{*} P<0.05$.

Growth of lung adenocarcinoma cells and transformation of normal ECS are inhibited by treatment with liposomal SOCS3. We first sought to evaluate the functional effects of liposomal SOCS3 on fully transformed cancer cells. A549 cells were thus treated with SOCS3-containing or empty liposomes. Cell number via CyQuant assay and cell confluence via IncuCyte were both significantly reduced by treatment with SOCS3-containing liposomes, as compared with control (no liposomes) and empty liposome-treated cells (Figure 5, A and B). This decrease correlated with decreased expression of the transcription factor and proto-oncogene forkhead box protein M1 (FoxM1) (Figure 5C), a cell cycle regulator that is upregulated in NSCLC cells (21). A significantly greater proportion of A549 cells expressed activated caspase-3/7 within the nucleus and surface annexin V (Figure 5, D and E, respectively) after treatment with SOCS3 liposomes than after treatment with empty liposomes, consistent with an increase in apoptosis; the frequency of annexin $\mathrm{V}^{+}$and caspase- $3 / 7^{+}$cells in response to SOCS3-containing liposomes was similar to that of cells treated with FasL. SOCS3 liposometreated cells also expressed higher amounts of Fas receptor (FasR) mRNA (Figure 5F), consistent with their enhanced apoptotic potential. Additionally, despite these proapoptotic actions, studies with the pan-caspase inhibitor Z-VAD-FMK demonstrated that the predominant mechanism by which SOCS3 liposomes reduced DNA content in A549 cells was via inhibition of proliferation (Figure 5G). Thus, the actions of liposomal SOCS3 on A549 cells largely paralleled those of SOCS3-containing natural AM-derived EVs (Figure 1F).

The observation that in vivo SOCS3 secretion during mutant Kras tumorigenesis declined as early as 4 weeks - hence preceding the development of visible tumors (Figure $2 \mathrm{~B}$ ) - suggested that its deficiency might facilitate malignant transformation. We therefore sought next to evaluate the capacity of SOCS3 
Table 2. Synthetic liposome physical characteristics

\begin{tabular}{cccc}
\hline Liposome & Size $(\mathbf{n m})$ & PDI & ZP \\
\hline Empty & $103 \pm 1$ & $0.21 \pm 0.01$ & $-15 \pm 8$ \\
SOCS3 & $124 \pm 33$ & $0.20 \pm 0.02$ & $-14 \pm 23$ \\
PDI, polydispersity index; zeta potential, ZP. & & \\
\hline
\end{tabular}

liposomes to restrain the process of malignant transformation itself. This was examined in a normal AEC line (RLE-6TN) engineered to express mutant KRAS $(\mathrm{G} 12 \mathrm{~V})$ and treated with cigarette smoke extract (CSE), by measuring anchorage-independent cell growth in soft agar (Figure $5 \mathrm{H}$ ). Neither mutant KRAS expression alone nor CSE alone was sufficient to induce EC transformation (data not shown). However, a 1-hour exposure to 3\% CSE daily for 7 consecutive days (intended to model the intermittent nature of cigarette smoking) resulted in colony growth of mutant KRAS cells but not of WT cells (Figure $5 \mathrm{H}$ ). Addition of SOCS3-containing liposomes, but not empty liposomes, concurrently with each dose of CSE significantly inhibited colony growth in KRAS mutant cells (Figure $5 \mathrm{H}$ ). This demonstrates that exogenously acquired SOCS3 inhibits EC transformation and supports the possibility that early loss of this acquired suppressor may contribute to tumorigenesis.

SOCS3 liposomes fail to promote a protumorigenic M2 phenotype in AMs. Having demonstrated that the actions of liposomal SOCS3 on ECs are tumor suppressive, we next examined its effects on the M1-M2 polarization status of AMs themselves. We utilized immortalized primary mouse AMs, treated them with empty or SOCS3 liposomes with or without agents that polarize toward M1 (IFN- $\gamma$ ) or M2 (IL-4/13) phenotypes, and measured expression of M1- (antitumor) and M2-associated (protumor) genes. Treatment of AMs with SOCS3 liposomes had no effect on the IFN- $\gamma$-induced expression of M1-associated inducible NOS (iNOS) (Supplemental Figure 2A) but potentiated that of the potent antitumor cytokine IL-12 (Supplemental Figure 2B). At the same time, SOCS3 liposomes significantly reduced the IL-4/13-induced expression of M2-associated TGF- $\beta$ and IL-10 (Supplemental Figure 2, C and D). We also examined the effects of liposomal SOCS3 on primary AMs harvested at 16 weeks after SPC-adenoviral infection from both WT and Kras mutant mice. As compared with treatment with empty liposomes, treatment with SOCS3 liposomes had no significant effect on expression of any of the M1 or M2 genes in AMs from Kras mutant mice (Supplemental Figure 2, E-H). Thus, SOCS3 liposomes did not — under any of the experimental conditions tested - exert any effects on AM cytokine profiles that would be considered protumorigenic.

SOCS3 liposomes inhibit tumor growth in a lung tumor xenograft model. In order to seek proof of concept that SOCS3 liposomes can exert antitumor effects in vivo, we performed s.c. A549 cell xenografts in athymic nude mice (Figure 6A). Once tumors became visible and palpable at about 4 weeks, mice were randomized to receive 2 intratumoral injections of either empty or SOCS3 liposomes on each of 6 occasions over the subsequent 2 weeks; tumor size was measured and tumor volume was calculated at each of these time points (Figure 6A). Tumors treated with empty liposomes exhibited steady growth over the 2-week follow-up period, whereas those treated with SOCS3 liposomes failed to increase their growth (Figure 6, B and C). Immunofluorescence microscopic analysis of tumor sections revealed decreased overall STAT3 phosphorylation following treatment with SOCS3 as compared with empty liposomes (Figure 6D). This was accompanied by a decreased proportion of nuclear-associated pSTAT3 (solid arrows) relative to cytoplasmic pSTAT3 (open arrowheads) in the SOCS3 liposome-treated group (Figure 6D). Additionally, histopathological analysis revealed an increase in necrosis in tumors treated with SOCS3 liposomes (Figure 6E). Taken together, these in vivo data reflect similar biological actions as those observed with in vitro liposome administration (Figure 4D).

\section{Discussion}

A substantial body of research in cancer of the lung and other organs has characterized and highlighted the protumorigenic potential of tumor-associated macrophages $(22,23)$. By contrast, the role of AMs - a resident population of macrophages present in the pulmonary alveolar space and airways prior to the development of lung cancer - is less well understood. In this study, we have uncovered a previously unrecognized role for secretion of SOCS3 within EVs by resident AMs as a functional inhibitor of tumor cells, which, however, can be sabotaged by the tumor microenvironment. Although EVs derived from tumor cells themselves 

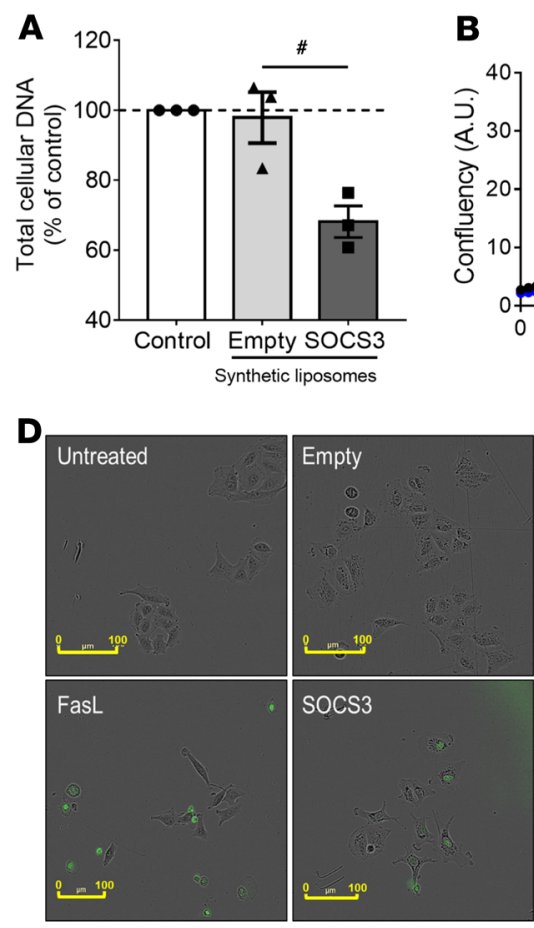

$\mathbf{E}$
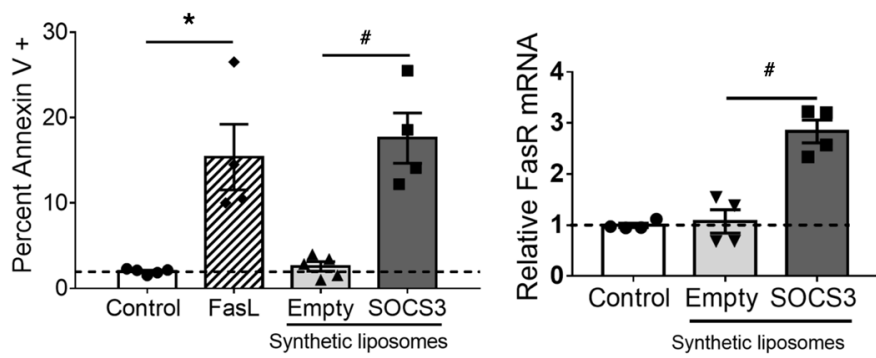

G

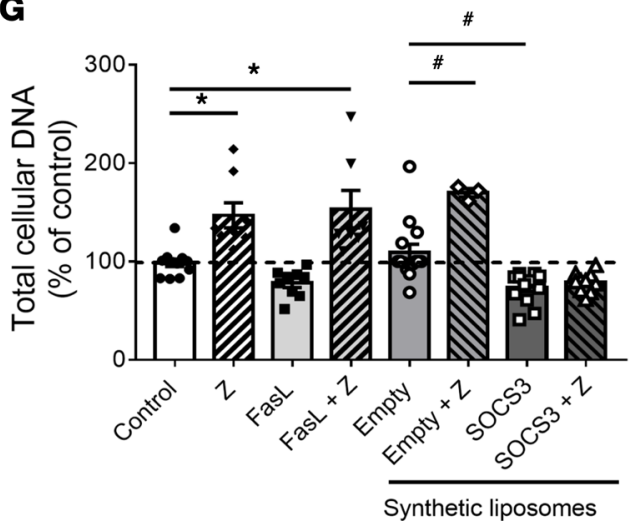

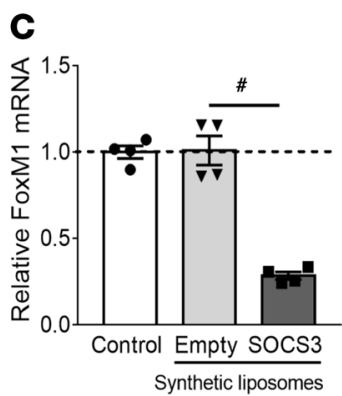

$\mathbf{F}$

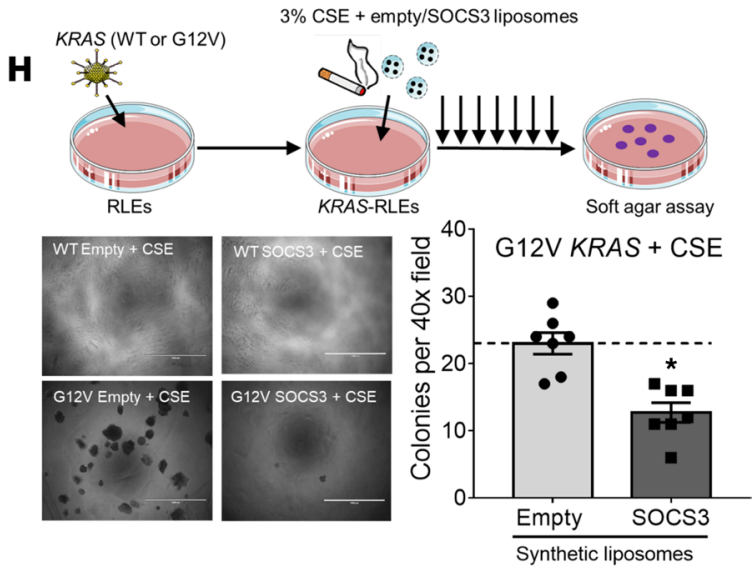

Figure 5. SOCS3 liposomes inhibit lung tumor cell function and epithelial cell transformation. (A) A549 cells were incubated with liposomes containing $10 \mathrm{ng} \mathrm{SOCS3}$ or an equal volume of liposomes containing PBS alone (empty) for $72 \mathrm{~h}$ and analyzed by CyQuant cell proliferation assay measuring total cellular DNA ( $n=3$ separate experiments). (B) One thousand A549 cells were plated per well of a 96 -well tissue culture plate and were incubated with empty or SOCS3 liposomes for $72 \mathrm{~h}$. Cellular confluence was measured using an IncuCyte Live Cell Analysis system. Data points represent the mean of quadruplicate measurements of confluency, expressed as AU per well, and are representative of triplicate wells per condition. (C) A549 cells were treated with empty or SOCS3 liposomes for $24 \mathrm{~h}$ and analyzed for FoxM1 mRNA expression by qPCR ( $n=3$ separate experiments). (D) Representative micrographs of caspase-3/7-positive cells after liposome treatment. Concurrent with the confluence assay in B, A549 cells were incubated with IncuCyte caspase-3/7 apoptosis assay reagent and empty or SOCS3 liposomes. Fluorescence micrographs are representative of 3 triplicate wells per treatment. Scale bar: $100 \mu \mathrm{m}$. (E) A549 cells were treated with empty or SOCS3 liposomes or FasL (as a positive control) for $24 \mathrm{~h}$, and annexin $\mathrm{V}^{+}$cells determined via flow cytometry ( $n=4-5$ replicate samples). (F) A549 cells were treated with empty or SOCS3 liposomes for 24 $\mathrm{h}$ and analyzed for relative FasR mRNA expression by qPCR ( $n=4$ separate experiments). (C) A549 cells were treated with FasL, empty liposomes, or SOCS3 liposomes for $24 \mathrm{~h}$, with or without the pan-caspase inhibitor Z-VAD-FMK (Z), and cell proliferation was determined by CyQuant assay ( $n$ = 5-14 replicates from 3 separate experiments). (H) Top: Schematic depiction of CSE transformation protocol for WT or G12V-RLE cells. A total of $0.5 \times 10^{6}$ RLE-6TN cells were incubated with either WT or G12V retrovirus for $24 \mathrm{~h}$. Cells were washed and selected with puromycin for an additional 48-72 h. Surviving cells were passaged an additional 2 times before use in experiments. For CSE transformation, cells were plated at a concentration of 10,000 cells/well of a 6 -well tissue culture dish and treated with $3 \%$ CSE, along with empty or SOCS3 liposomes, for $1 \mathrm{~h}$ daily for 7 consecutive days. They were then cultured in equal numbers in a soft agar colony assay for an additional 7 days before colonies were enumerated. Bottom left: Representative micrographs of CSE-treated WT or G12V RLEs within a soft agar colony assay. Photographs were taken at 40x magnification. Scale bar: $1000 \mu \mathrm{m}$. Bottom right: Data represents mean colony counts from 7 separate $40 \times$ fields per treatment from 3 separate experiments. Student's 2-tailed $t$ test was used for comparison of 2 groups (A, C, F, and $\mathbf{H}$ ), or 1-way ANOVA with Bonferroni's post hoc analysis was used for multiple comparisons (E and $\mathbf{G}) ;{ }^{*} P<0.05$ or $\# P<0.05$ represents comparison to control or empty liposomes, respectively. 
A

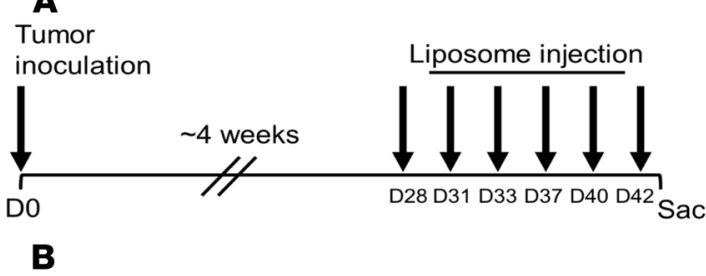

B

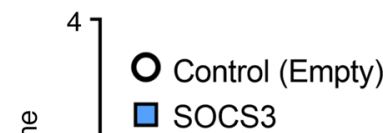

D

Days post $\mathrm{A} 549$ inoculation
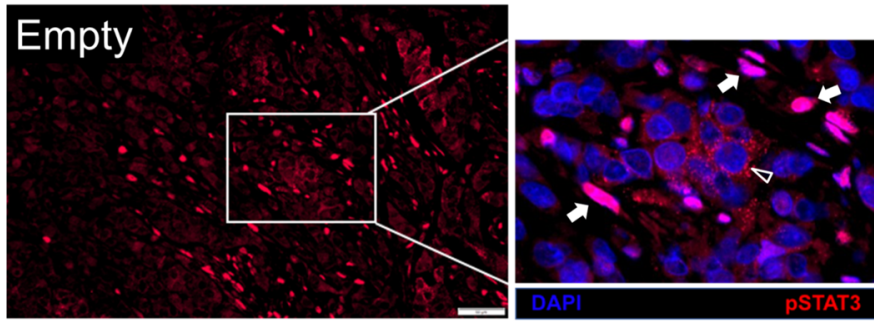

\section{SOCS3}
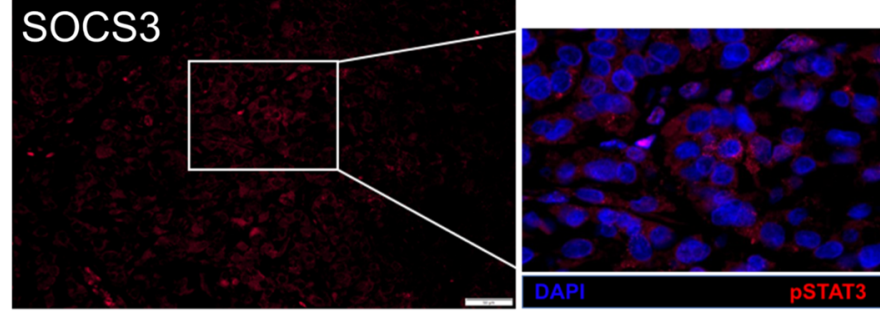

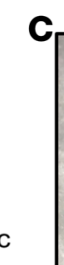

$\mathbf{C}$

Empty liposomes

Empty liposomes

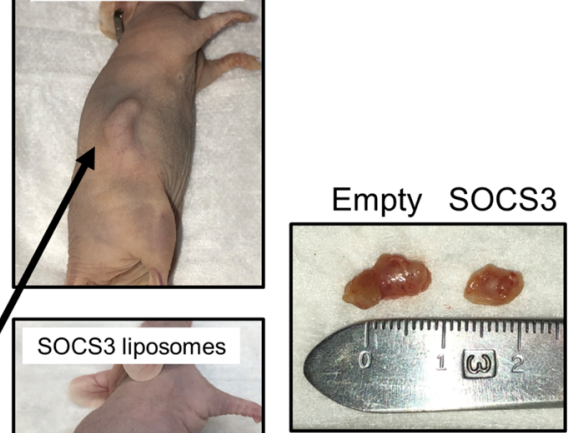

Day 42
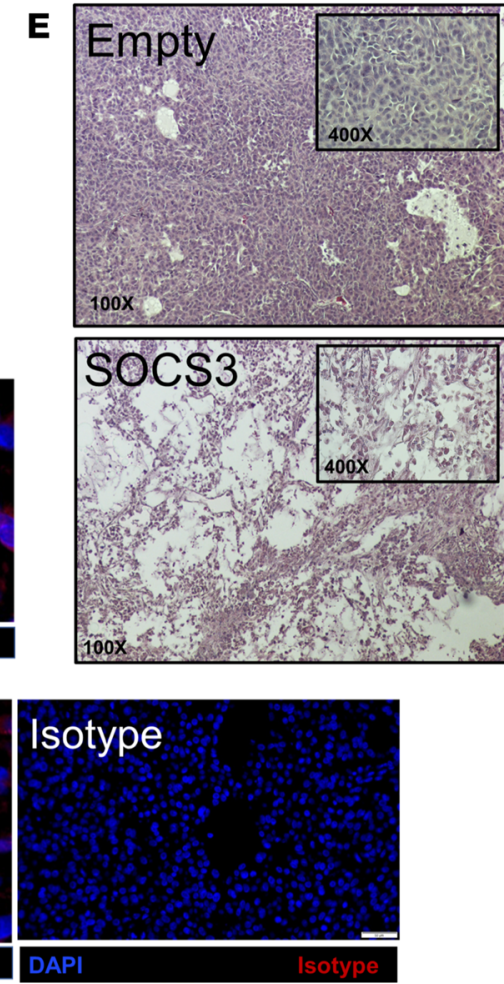

Figure 6. Intratumoral administration of SOCS3 liposomes inhibits tumor growth in nude mice. (A) Experimental scheme: $1 \times 10^{6} \mathrm{~A} 549$ cells were injected s.c. in athymic nude mice at day 0 . After palpable tumors were observed at $\sim 28$ days, mice were randomized into 2 groups and treated s.c. with liposomes containing $10 \mathrm{ng}$ SOCS3 or an equal volume of liposomes containing PBS (at 2 sites per tumor) in $50 \mu$ I PBS approximately every other day for a total of 6 treatments. Tumor measurements were taken 2 days prior to the first liposome injection and on every subsequent injection day. (B) Mean tumor volumes from empty and SOCS3 treated mice ( $n=4-7$ mice per group). (C) Images of representative whole tumors from mice treated with empty and SOCS3 liposomes. (D) Representative images of isotype, pSTAT3 and DAPI labeled tumors sections. Solid arrows represent nuclear-associated pSTAT3; open arrowheads represent cytoplasmic-associated pSTAT3. (E) Representative H\&E-stained tumors from A549 tumor-bearing mice treated with empty and SOCS3 liposomes. One-way ANOVA with Bonferroni's post hoc analysis was used for multiple comparisons.

have been implicated in various aspects of tumorigenesis or metastasis (20), the role of EVs derived from resident AMs in tumor development has not previously been investigated. The loss of functional SOCS3 within ECs themselves - owing to mutation or epigenetic downregulation - is well established to favor tumorigenesis via a variety of mechanisms $(3,24,25)$. However, to our knowledge, there has never been any previous 
indication that AMs can serve as an exogenous source of a tumor suppressor within the lung. Our findings reveal that, under normal circumstances, SOCS3 acquired from AM EVs is functionally able to restrain epithelial tumorigenesis. The deficiency of SOCS3 at the mucosal surface due to impaired AM secretion that occurs during lung tumor initiation may both contribute to EC transformation and confer growth and survival advantages to mature tumor cells.

Our data demonstrate that the SOCS3 secretory defect observed in AMs from tumor-bearing lungs is not explained by decreases in AM expression of SOCS3 or total EV release, but rather reflects diminished packaging of cellular SOCS3 within secreted EVs. This is a cell-intrinsic defect that persists in AMs removed from the tumor-bearing lung, as evidenced by the data in Figure 3, G-I. It is also notable that SOCS3 secretion in the tumor-bearing lungs is diminished, despite - as expected - high BALF levels of prostaglandin $\mathrm{E}_{2}$ (data not shown), which we have previously established to be a potent SOCS3 secretagogue in AMs (11). Molecular mechanisms for selective protein cargo packaging within EVs are poorly understood, and it remains to be determined how such a mechanism regulating SOCS3 packaging in AMs might be perturbed by their residence in the tumor-bearing lung. It is also unknown whether this SOCS3 secretory defect extends to vesicular packaging of other cargo molecules. The defect in SOCS3 secretion by AMs was identified in a commonly employed mouse model of adenocarcinoma driven by mutant Kras. While the genetic profile of our patients' tumors was not determined in this study, it is likely that they manifested a variety of mutations; any relationship between specific tumor mutations and such a SOCS3 secretory defect remains to be determined. Finally, the mechanism(s) underlying this secretory defect in AMs, and whether it reflects the direct inhibitory actions of molecules in the alveolar milieu of the tumor-bearing lung, also remains to be defined.

Given the important role of STAT activation in promoting proliferation and survival of tumor cells and, therefore, of maintaining SOCS3 expression in opposing these STAT-dependent responses, a variety of approaches to modulate the STAT-SOCS3 axis have been explored. These include pharmacologic inhibitors of STAT activation (e.g., ruxolitinib) as well as exogenous expression of SOCS3 via viral vectors (7, $24,25)$ and administration of SOCS peptides $(8,26)$. STAT inhibitors, while promising in hematological cancers such as multiple myeloma (27), failed in clinical drug trials of solid tumors owing to toxicity and tumor resistance due to pathway compensation (28). Viral transduction of SOCS3 expression has not yet been tested in humans in vivo; therefore, its effectiveness and safety at this point are unknown. SOCS3 peptides have shown promise in xenograft models of squamous cell carcinoma and breast cancer $(8,26)$, but therapeutic peptides have a variety of disadvantages, including poor membrane permeability, rapid clearance, and metabolic instability (29).

The tumor suppressor PTEN has been reported to be transferred between cell types via EVs in nontumor models (30). Furthermore, recent reports describe efforts to engineer the expression of miRNAs with tumor-suppressive actions within cell-derived EVs $(30,31)$. We report here that natural EVs released by AMs and containing the tumor suppressor SOCS3 protein inhibited diverse tumor cell functions. The prospect of treatment with natural EVs poses a variety of immunologic, logistical, and regulatory challenges. To circumvent these limitations, we engineered a synthetic mimic thereof, namely phospholipid liposomes containing recombinant SOCS3 as their sole cargo. These inhibited malignant transformation of normal AECs, as well as proliferation and survival of an established AEC-derived cancer cell line both in vitro and in a xenograft model of lung tumor growth. Homogeneous liposomes of uniform phospholipid and SOCS3 cargo content and size can be readily generated to large scale, are easy to modify, are stable in vivo, and have the potential to be delivered to the lung via aerosolization (32); they thereby closely mimic the natural delivery of SOCS3 by AMs within the alveolar/airway microenvironment. Additionally, SOCS3 can inhibit additional signaling pathways implicated in tumorigenesis besides JAK/STAT (6). Indeed, we speculate that this may help to explain the observation that the functional impact of SOCS3 liposomes on endpoints such as proliferation and apoptosis exceeded the degree to which they inhibited STAT3 activation. For all of these reasons, we suggest that SOCS3 liposomes may represent a treatment strategy that is both more stable and exerts a broader inhibitory potential than STAT inhibitors, thus reducing the likelihood of pathway resistance mechanisms by the tumor.

In tumor environments, macrophages polarize toward an alternatively activated or M2 phenotype that promotes tumorigenesis via a variety of mechanisms (14). If administration of SOCS3 liposomes is ever to be considered for in vivo prevention/treatment of lung cancer or other tumors, it is important to ensure that SOCS3 liposomes do not augment the protumor effects of AMs. Indeed, we saw no evidence that SOCS3 
liposomes either promoted the polarization of AMs toward an M2 phenotype or diminished their M1/M2 balance. Besides macrophages, a number of other structural or tumor-associated immune cells (i.e., fibroblasts, ECs, CD8 ${ }^{+} \mathrm{T}$ cells, Tregs, and myeloid-derived suppressor cells) might also be expected to take up liposomes and, as a consequence, manifest either pro- or antitumor effects. Since tumor cells have been shown to exhibit enhanced EV uptake as compared with normal cells (33), consistent with what we observed in this study (Figure 1A), it is likely that the tumor cells will be the main target for liposome uptake and actions. Nevertheless, the effects of SOCS3 liposomes on other cellular constituents of tumors are deserving of future study. These will best be addressed by utilizing tumor models within the lung microenvironment, rather than xenografts.

In conclusion, we have uncovered a previously unrecognized mechanism by which lung tumor progression is facilitated by loss of AM-secreted SOCS3. We have also provided proof-of-concept data confirming that rescue of this normal brake on tumorigenesis via administration of SOCS3 liposomes can inhibit tumor formation in vitro and in vivo. Future studies will focus on identifying the molecular mechanism by which elaboration of this natural tumor suppressor is dysregulated during lung tumorigenesis and whether restoration of SOCS3 via intrapulmonary administration of synthetic liposomes can serve as a therapeutic strategy to treat or prevent NSCLC in mouse models.

\section{Methods}

Rat and mouse AM isolation. Primary AMs were collected from rat or mouse lung lavage fluid and plated in serum-free RPMI 1640 with L-glutamine (Thermo Fisher Scientific) at a concentration of $1 \times 10^{6}$ cells/ml, as previously described (12).

EV isolation. CM from primary rat AMs was collected after 24-hour culture, and EVs were isolated as previously described (11). Briefly, CM was sequentially centrifuged at $500 \mathrm{~g}$ for 10 minutes, $2500 \mathrm{~g}$ for 12 minutes, and 17,000 $\mathrm{g}$ for 160 minutes to remove dead cells/debris and apoptotic bodies and to pellet EVs, respectively. EVs were resuspended in $200 \mu 1$ serum-free RPMI and counted using flow cytometry as previously described (11). When administered to ECs in vitro, EVs were dosed at approximately 16 EVs per 1 $\mathrm{EC}$, which is within the range that is typically employed in such experiments.

Cell lines and AM immortalization. The human lung adenocarcinoma cell line A549 and normal rat lung EC lines (RLE-6TN and L2) were purchased from the ATCC and were cultured in either RPMI (A549) or Ham's F-12K (Kaign's modification) (Thermo Fisher Scientific) with 10\% FCS (HyClone) and penicillin-streptomycin (Thermo Fisher Scientific) at $37^{\circ} \mathrm{C}$ and $5 \% \mathrm{CO}_{2}$. Primary mouse AMs were immortalized by infection with the $\mathrm{J} 2$ retrovirus, as previously described (34). Briefly, AMs were harvested from the lungs of mice and cultured overnight in RPMI with $10 \%$ FCS and penicillin-streptomycin. Cells were then washed and cultured in $\mathrm{J} 2$ retrovirus supernatants for 1 day. They were next washed and cultured in RPMI with 5\% FCS, HEPES (Thermo Fisher Scientific), and penicillin-streptomycin for 1 day. This process was repeated once more, and cells were then cultured in RPMI with 5\% FCS, HEPES until immortalization was observed.

EV uptake assay in A549 cells. Both L2 cells (a normal rat lung EC line) and A549 cells (10,000/well of a 96-well tissue culture plate) were incubated with PKH26-labeled AM EVs at a ratio of 16 EVs per 1 EC for 1 hour. The cells were washed with PBS (Thermo Fisher Scientific) twice, trypsinized, and analyzed for green fluorescence by flow cytometry as previously described (35). An LSRFortessa flow cytometer from BD Biosciences was used, and data were analyzed with FlowJo 10.5.0 software (Tree Star Inc.).

Western blot. A549 cells were harvested, lysed, and analyzed by Western blot as previously described (11, 12). Monoclonal antibodies for SOCS3 (SO1, Abcam), KRAS G12V (D2H12, Cell Signaling Technology) and $\beta$-actin (AC-74, MilliporeSigma) were used.

Proliferation assay. A549 cells were adhered overnight in complete RPMI medium in a 96-well plate at a concentration of 1,000 cells/well. Cells were subsequently serum starved in RPMI containing 2\% FCS and penicillin-streptomycin for 24 hours before incubation with empty liposomes, SOCS3-containing liposomes, or AM EVs for 72 hours. For inhibition of caspase activity, cells were pretreated with $20 \mu \mathrm{M} Z$-VAD-FMK (InvivoGen) for 30 minutes prior to vesicle treatment. Cell proliferation was determined using the CyQuant proliferation assay (Thermo Fisher Scientific) for DNA binding per manufacturer's instructions.

Apoptosis assay. A549 cells were adhered overnight in complete RPMI medium in 12-well tissue culture plates at a concentration of 10,000 cells/well. Cells were treated with empty liposomes, SOCS3-containing liposomes, AM EVs, or FasL (CH11, MilliporeSigma, as a positive control) for 24 hours. Cells were subsequently stained with annexin V Pacific Blue antibody (BioLegend), and annexin $\mathrm{V}^{+}$(apoptotic) cells were enumerated using flow cytometry. 
Human BALF sample acquisition. BALF samples were acquired from a total of 22 patients undergoing diagnostic bronchoscopic biopsy at the University of Michigan Hospital Medical Procedure Unit for suspected lung cancer who were subsequently determined to have NSCLC. A total of 12 BALF samples were also collected from healthy volunteers without lung disease or suspicion of malignancy. Demographic characteristics of both groups of subjects are presented in Figure 2B. For both groups of subjects, BALF samples were collected and treated as protocolized research specimens with the same instillation volume $(180 \mathrm{ml})$.

Animals. Pathogen-free 8-week-old female Wistar rats (Charles River Laboratories), 6- to 8-week-old LSL-Kras ${ }^{\mathrm{G} 12 \mathrm{D}}$ mice, WT littermate controls (mixed sex), and female athymic Nu/J mice (all from the Jackson Laboratory) were used. Mice and rats were maintained at the University of Michigan Unit for Laboratory Animal Medicine. Mutant Kras expression was initiated by oropharyngeal administration of Ad5CMV-Cre or Ad5SPC-Cre $\left(2.5 \times 10^{7} \mathrm{pfu}\right.$ and $2 \times 10^{7} \mathrm{pfu}$, respectively). Mice were sacrificed at 2, 4, 8, 10, 12, or 16 weeks after adenoviral infection.

SOCS3 ELISA. Human and mouse BALF was harvested and processed for ELISA as previously described (12). Samples were sonicated to disrupt EVs and were analyzed using a human or rat SOCS3 ELISA kit (Cloud-Clone Corp.).

Flow cytometric analysis of resident AMs. Resident AMs were analyzed by flow cytometry using antibodies for CD11c-PerC/PCy5.5 (N418, BioLegend), CD11b-FITC (M1/70, BD Pharmingen), and Siglec-F-PE (E5-2440, BD Pharmingen). Resident AM polarization status was analyzed using antibodies for iNOS-APC (clone CXNFT, M1 phenotype) and CD206-V450 (clone MR6F3, M2 phenotype) (Thermo Fisher Scientific).

RNA isolation and quantitative PCR ( $q P C R$ ) analysis. RNA was extracted from A549 cells, immortalized AMs, and primary AMs using QIAGEN columns according to manufacturer's instructions and converted to cDNA via reverse transcription. FoxM1, cyclin A1, cyclin B1, cyclin E1, FasR, G12V KRAS, SOCS3, iNOS, IL-12, TGF- $\beta$, and IL-10 mRNA levels were assessed using SYBR Green (Applied Biosystems) using an ABI Prism 7300 thermocycler (Applied Biosystems). Relative gene expression was determined by the $\triangle \mathrm{CT}$ method, with GAPDH or $\beta$-actin as a reference gene (primer sequences listed in Supplemental Table 1).

Generation of recombinant SOCS3 protein. Mouse SOCS3 protein was cloned in the bacterial expression vector pMCSG7, followed by transformation in Rosetta 2 competent cells (Millipore Sigma). Expression of SOCS3 protein was induced with isopropyl $\beta$-D-1-thiogalactopyranoside and enriched using Ni-NTA affinity columns, followed by purification using fast protein liquid chromatography. Because mouse and human SOCS3 proteins are $97 \%$ homologous overall and 100\% homologous within the functionally critical kinase inhibitory region, liposomes encapsulating recombinant mouse SOCS3 could be used for treatment of both mouse and human target cells.

Generation of synthetic SOCS3 liposomes. All liposomes were produced by extrusion after thin film hydration. Briefly, phospholipid mixtures of 1:1 (molar ratio) 1,2-dioleoyl-sn-glycero-3-phosphocholine (DOPC, Avanti Polar Lipids) and 1,2-dioleoyl-sn-glycero-3-phospho-(1'-rac-glycerol) (sodium salt; DOPG, Avanti Polar Lipids) were dried under nitrogen gas to produce thin phospholipid films and placed in a vacuum oven for a minimum of 2 hours. PBS, with or without recombinant SOCS3 protein $(3.5 \mu \mathrm{g} / \mathrm{ml})$, was added to the dried films and intermittently vortexed over a period of $\sim 40$ minutes to produce multilamellar vesicles, followed by serial extrusion (mini extruder, Avanti Polar Lipids) following the manufacturer's instructions to produce unilamellar liposomes with a diameter of approximately $100 \mathrm{~nm}$ (selected to mimic the mean diameter determined by nanotracker analysis for natural AM-derived EVs). The produced liposomes were ultracentrifuged (Airfuge, Beckman Coulter) at 200,000 $\mathrm{g}$ for 30 minutes at room temperature to remove unloaded SOCS3 protein contained in the supernatant. The pellets containing liposomes were suspended in $0.22 \mu \mathrm{m}$ filtered PBS and stored at $4^{\circ} \mathrm{C}$ until use. The hydrodynamic sizes and zeta potentials of the liposomes were measured after ultracentrifugation by dynamic light scattering with a Zetasizer ZSP (Malvern Panalytical) using PBS as a dispersant.

IncuCyte assay of cell confluence and caspase 3/7 activity. One thousand A549 cells were seeded per well of a 96-well tissue culture plate. Cells were cultured in RPMI plus 5\% FCS and penicillin-streptomycin, and treated with RPMI alone, empty liposomes, SOCS3 liposomes, or FasL in triplicate wells. At the beginning of the assay, IncuCyte caspase 3/7 green apoptosis reagent (Essen Bioscience) was added to the medium per manufacturer's instructions. Cell confluence and caspase $3 / 7$ activity were analyzed using an IncuCyte Live Cell Analysis system (Essen Bioscience). Phase contrast and green fluorescence readings were taken every 2 hours for a total of 72 hours. 
Lentiviral G12V or WT KRAS expression in RLE-6TN cells. RLE-6TN cells were infected with pBABEpuro-KRAS (WT) or pBABE-puro-KRAS G12V (mutant) lentiviral vectors for 24 hours. For generation of stably expressing cell lines, cells were selected with puromycin (InVivoGen) for an additional 3 passages. Expression of either WT or G12V KRAS was confirmed using qPCR (primer sequences listed in Supplemental Table 1).

Transformation of RLE-6TN cells and soft agar assay. WT and G12V KRAS RLE-6TN cells were subjected to transformation by treating for 1 hour/day for a total of 7 days with 3\% CSE in F12K plus 10\% FBS, in the presence of either empty or SOCS3 liposomes. Twenty-four hours after the final CSE exposure, an equal number of cells from each group were harvested for soft agar assays. Soft agar assays were performed using the CytoSelect 96-well transformation assay (Cell BioLabs) per manufacturer instructions. Colonies were enumerated from an average of eight $40 \times$ fields by light microscopy.

Analysis of AM polarization. Immortalized AMs and primary AMs isolated from WT or LSL-Kras ${ }^{\mathrm{G} 12 \mathrm{D}}$ mice were cultured for 24 hours in RPMI plus $10 \%$ FCS containing either $10 \mathrm{ng} / \mathrm{ml}$ recombinant mouse IFN- $\gamma$ or $10 \mathrm{ng} / \mathrm{ml}$ each of both recombinant mouse IL- 4 and IL-13 to induce polarization to M1 or M2 macrophages, respectively. Cells were lysed and analyzed for M1- and M2-associated genes by qPCR.

A549 xenograft model in nude mice. Seven- to 8-week old athymic $\mathrm{Nu} / \mathrm{J}$ mice were injected s.c. with $1 \times 10^{6}$ A549 cells, and tumors were allowed to grow until visible to the naked eye, typically at approximately 4 weeks. Once tumors were established, mice were randomized into 2 groups (4-7 mice per group) for s.c. treatment with either empty liposomes or SOCS3 liposomes. Treatments consisted of injecting a bolus of liposomes on each of 2 opposite sides of the tumor just outside the tumor margin. Mice were treated 6 times over the subsequent 14 days, and tumor dimensions were measured prior to each treatment. Tumor volumes were calculated using the formula: volume $=\mathrm{xy}^{2} / 2$, where $\mathrm{x}$ and $\mathrm{y}$ represent measurements of 2 orthogonal diameters.

A549 tumor histopathology and immunofluorescent staining. A549 tumors were resected, fixed in 10\% formalin for 24 hours at $4^{\circ} \mathrm{C}$, and then processed and embedded in paraffin. For immunostaining of pSTAT3 within tumors, sections were cut at $4 \mu \mathrm{m}$, deparaffinized, hydrated, boiled in EDTA unmasking solution (Cell Signaling Technologies), blocked in 10\% donkey serum (MilliporeSigma) in Tris buffered saline with 0.05\% Tween (TTBS, Bio-Rad), and incubated with antibody against pSTAT3 (Tyr705; 1:50; Cell Signaling Technologies) overnight at $4^{\circ} \mathrm{C}$. Secondary antibody Cy3-conjugated anti-rabbit IgG (H+L) (Jackson ImmunoResearch) was applied for 1 hour at room temperature followed by DAPI counterstaining. Tumors were imaged using a 20× (UPlanSApo, 0.75 NA) objective of an Olympus BX53 with a motorized stage (Prior Scientific) and cellSens Dimension software (Olympus).

Statistics. Data are expressed as mean \pm SEM from at least 3 independent experiments unless noted otherwise in the figure legend. Data were analyzed using the Prism 5.0 statistical program from GraphPad software, using either 1-way ANOVA with Bonferroni's post hoc analysis or Student's 2-tailed $t$ test, as appropriate. Statistical significance was set at $P<0.05$. Significant values are labeled with an asterisk $\left({ }^{*}\right)$ or hash mark (\#) representing comparison with control or empty liposomes, respectively.

Study approval. Informed consent was obtained from each subject prior to sample collection in accordance with the Declaration of Helsinki and with approval of the University of Michigan Institutional Review Board. Animals were treated according to NIH guidelines for the use of experimental animals with the approval of the University of Michigan Committee for the Use and Care of Animals.

\section{Author contributions}

JMS designed, performed, and analyzed experiments and drafted the manuscript. LRP performed experiments and assisted with manuscript preparation. DJS assisted with experiments and provided experimental advice. JDB and KSP produced and optimized synthetic liposomes. RGDR performed IF staining and $\mathrm{H} \& \mathrm{E}$ staining. HO procured and processed patient BAL samples and performed experiments. JJM produced synthetic liposomes and edited the manuscript. VGK provided experimental advice and reviewed and edited the manuscript. RLZ assisted with IF analysis, provided experimental advice, and reviewed and edited the manuscript. VNL provided normal patient BAL and provided experimental advice. DAA performed patient bronchoscopies, provided experimental advice, and reviewed and edited the manuscript. MPG supervised the study, provided scientific insight, and reviewed and edited the manuscript. 


\section{Acknowledgments}

We thank Jeanne Stuckney and Krishnapriya Chinnaswamy from the University of Michigan Center for Structural Biology for their help with recombinant SOCS3 purification. We also thank David Beer for his input into the development of this project. The studies in this manuscript were supported by NIH grant R01 HL125555 (to MPG). JMS was supported by NIH T32 training grant HL 7749-23, Michigan Institute for Clinical \& Health Research (MICHR) grant UL1TR00043, and American Cancer Society grant PF-17-14301-TBG3. RGDR and RLZ were supported by NIH grant 2T32HL007749-26.

Address correspondence to: Marc Peters-Golden, 6301 Medical Science Research Building III, 1150 West Medical Center Drive, Ann Arbor, Michigan 48109-5642, USA. Phone: 734.936.5047; Email: petersm@umich.edu.

1. Ferrara R, et al. Hyperprogressive Disease in Patients With Advanced Non-Small Cell Lung Cancer Treated With PD-1/PD-L1 Inhibitors or With Single-Agent Chemotherapy. JAMA Oncol. 2018;4(11):1543-1552.

2. Karamouzis MV, Konstantinopoulos PA, Papavassiliou AG. The role of STATs in lung carcinogenesis: an emerging target for novel therapeutics. J Mol Med. 2007;85(5):427-436.

3. Li Y, Du H, Qin Y, Roberts J, Cummings OW, Yan C. Activation of the signal transducers and activators of the transcription 3 pathway in alveolar epithelial cells induces inflammation and adenocarcinomas in mouse lung. Cancer Res. 2007;67(18):8494-8503

4. $\mathrm{Qu} \mathrm{P}$, et al. Stat 3 downstream genes serve as biomarkers in human lung carcinomas and chronic obstructive pulmonary disease. Lung Cancer. 2009;63(3):341-347.

5. Chikuma S, Kanamori M, Mise-Omata S, Yoshimura A. Suppressors of cytokine signaling: Potential immune checkpoint molecules for cancer immunotherapy. Cancer Sci. 2017;108(4):574-580.

6. Inagaki-Ohara K, Kondo T, Ito M, Yoshimura A. SOCS, inflammation, and cancer. JAKSTAT. 2013;2(3):e24053.

7. Lin YC, et al. Adenovirus-mediated SOCS3 gene transfer inhibits the growth and enhances the radiosensitivity of human nonsmall cell lung cancer cells. Oncol Rep. 2010;24(6):1605-1612.

8. Madonna S, et al. SOCS3 inhibits the pathological effects of IL-22 in non-melanoma skin tumor-derived keratinocytes. Oncotarget. 2017;8(15):24652-24667.

9. Cho HC, Lai CY, Shao LE, Yu J. Identification of tumorigenic cells in Kras(G12D)-induced lung adenocarcinoma. Cancer Res. 2011;71(23):7250-7258.

10. Hanna JM, Onaitis MW. Cell of origin of lung cancer. J Carcinog. 2013;12:6.

11. Bourdonnay E, et al. Transcellular delivery of vesicular SOCS proteins from macrophages to epithelial cells blunts inflammatory signaling. J Exp Med. 2015;212(5):729-742.

12. Speth JM, et al. Alveolar Epithelial Cell-Derived Prostaglandin E2 Serves as a Request Signal for Macrophage Secretion of Suppressor of Cytokine Signaling 3 during Innate Inflammation. J Immunol. 2016;196(12):5112-5120.

13. Pouniotis DS, Plebanski M, Apostolopoulos V, McDonald CF. Alveolar macrophage function is altered in patients with lung cancer. Clin Exp Immunol. 2006;143(2):363-372.

14. Almatroodi SA, McDonald CF, Pouniotis DS. Alveolar Macrophage Polarisation in Lung Cancer. Lung Cancer Int. 2014;2014:721087.

15. Sutherland KD, Song JY, Kwon MC, Proost N, Zevenhoven J, Berns A. Multiple cells-of-origin of mutant K-Ras-induced mouse lung adenocarcinoma. Proc Natl Acad Sci USA. 2014;111(13):4952-4957.

16. Lewis KM, et al. Small-molecule targeting of signal transducer and activator of transcription (STAT) 3 to treat non-small cell lung cancer. Lung Cancer. 2015;90(2):182-190.

17. Zhang S, Guo D, Jiang L, Zhang Q, Qiu X, Wang E. SOCS3 inhibiting migration of A549 cells correlates with PYK2 signaling in vitro. BMC Cancer. 2008;8:150

18. He B, et al. SOCS-3 is frequently silenced by hypermethylation and suppresses cell growth in human lung cancer. Proc Natl Acad Sci USA. 2003;100(24):14133-14138.

19. DuPage M, Dooley AL, Jacks T. Conditional mouse lung cancer models using adenoviral or lentiviral delivery of Cre recombinase. Nat Protoc. 2009;4(7):1064-1072.

20. Tippimanchai DD, et al. Adenoviral vectors transduce alveolar macrophages in lung cancer models. Oncoimmunology. 2018;7(6):e1438105.

21. Kim IM, et al. The Forkhead Box $\mathrm{m} 1$ transcription factor stimulates the proliferation of tumor cells during development of lung cancer. Cancer Res. 2006;66(4):2153-2161.

22. Fridlender ZG, Albelda SM. Modifying tumor-associated macrophages: An important adjunct to immunotherapy. Oncoimmunology. 2013;2(12):e26620.

23. Wang R, et al. Tumor-associated macrophages provide a suitable microenvironment for non-small lung cancer invasion and progression. Lung Cancer. 2011;74(2):188-196.

24. Cooper JC, Shi M, Chueh FY, Venkitachalam S, Yu CL. Enforced SOCS1 and SOCS3 expression attenuates Lck-mediated cellular transformation. Int J Oncol. 2010;36(5):1201-1208.

25. Zhang S, Wang W, Wang E, Qiu X. SOCS3 expression is inversely correlated with Pyk2 in non-small cell lung cancer and exogenous SOCS3 inhibits proliferation and invasion of A549 cells. Pathology. 2012;44(5):434-440.

26. La Manna S, et al. Mimetics of suppressor of cytokine signaling 3: Novel potential therapeutics in triple breast cancer. Int J Cancer. 2018;143(9):2177-2186.

27. Lin J, Hu Y, Zhao JJ.Repression of Multiple Myeloma Cell Growth In Vivo by Single-wall Carbon Nanotube (SWCNT)-delivered MALAT1 Antisense Oligos. J Vis Exp. 2018(142).

28. Wong ALA, Hirpara JL, Pervaiz S, Eu JQ, Sethi G, Goh BC. Do STAT3 inhibitors have potential in the future for cancer therapy? 
Expert Opin Investig Drugs. 2017;26(8):883-887.

29. Marqus S, Pirogova E, Piva TJ. Evaluation of the use of therapeutic peptides for cancer treatment. J Biomed Sci. 2017;24(1):21.

30. Almanza G, Rodvold JJ, Tsui B, Jepsen K, Carter H, Zanetti M. Extracellular vesicles produced in B cells deliver tumor suppressor miR-335 to breast cancer cells disrupting oncogenic programming in vitro and in vivo. Sci Rep. 2018;8(1):17581.

31. O'Brien KP, et al. Employing mesenchymal stem cells to support tumor-targeted delivery of extracellular vesicle (EV)-encapsulated microRNA-379. Oncogene. 2018;37(16):2137-2149.

32. Rudokas M, Najlah M, Alhnan MA, Elhissi A. Liposome Delivery Systems for Inhalation: A Critical Review Highlighting Formulation Issues and Anticancer Applications. Med Princ Pract. 2016;25 Suppl 2:60-72.

33. Nakase I, Kobayashi NB, Takatani-Nakase T, Yoshida T. Active macropinocytosis induction by stimulation of epidermal growth factor receptor and oncogenic Ras expression potentiates cellular uptake efficacy of exosomes. Sci Rep. 2015;5:10300.

34. Zhou H, Imrich A, Kobzik L. Characterization of immortalized MARCO and SR-AI/II-deficient murine alveolar macrophage cell lines. Part Fibre Toxicol. 2008;5:7.

35. Schneider DJ, Speth JM, Penke LR, Wettlaufer SH, Swanson JA, Peters-Golden M. Mechanisms and modulation of microvesicle uptake in a model of alveolar cell communication. J Biol Chem. 2017;292(51):20897-20910. 